\title{
Emission and Laser Absorption Spectroscopy of Flat Flames in Aluminum Suspensions
}

\author{
Michael Soo $^{\mathrm{a}}$, Samuel Goroshin ${ }^{\mathrm{a}}$, Nick Glumac ${ }^{\mathrm{b}}$, Keishi Kumashiro ${ }^{\mathrm{a}}$, James Vickery ${ }^{\mathrm{a}}$, \\ David L. Frost ${ }^{\mathrm{a}}$, Jeffrey M. Bergthorson ${ }^{\mathrm{a}}$ \\ ${ }^{a}$ McGill University \\ Department of Mechanical Engineering \\ 817 Sherbrooke West \\ Montréal, QC H3A 0C3, Canada \\ ${ }^{\mathrm{b}}$ University of Illinois at Urbana-Champaign \\ Mechanical Science and Engineering \\ 1206 West Green Street \\ Urbana, IL 61801, USA
}

*Corresponding author: Michael Soo, michael.soo@mail.mcgill.ca

\begin{abstract}
Imaging emission spectroscopy, spatially resolved laser-absorption spectroscopy, and particle image velocimetry (PIV) are applied to a flat flame stabilized in a suspension of micron-sized aluminum. The results from the combination of diagnostics are used to infer the combustion regime of the particles and to estimate the characteristic combustion time of the suspension. It is observed that the reaction zone of the flame in stoichiometric aluminumair suspensions exhibits strong self-reversal of the atomic aluminum emission lines. These lines also exhibit high optical depths in both emission and absorption spectroscopy. The strong self-reversal and high optical depths indicate high concentrations of aluminum vapor within the reaction zone of the flame at multiple temperatures. These features provide evidence of the formation of vapor-phase micro-diffusion flames around the individual particles in the suspension. In aluminum-methane-air flames, the lack of self-reversal and lower optical depths of the aluminum atomic lines indicate the absence of vapor-phase micro-diffusion flames, and point to a more heterogeneous, and likely kinetically-controlled, particle combustion regime. The reaction zone thickness is estimated from the spatially resolved profiles of aluminum resonance lines in both absorption and emission through the flame. The emission measurements yield a reaction zone thickness on the order of $1.7 \pm 0.3 \mathrm{~mm}$ in aluminum-air flames, and the absorption measurements yield a thickness on the order of $2.3 \pm 0.5$. It is demonstrated that the combination of the combustion zone thickness measurement, flame temperatures determined from molecular $\mathrm{AlO}$ emission spectra, and particle velocity measurements from the PIV diagnostic permits an estimation of the burning time in the suspension. The burning time in stoichiometric aluminum-air suspensions using the suite of diagnostics is estimated to be on the order of 0.7 milliseconds.
\end{abstract}

Keywords: aluminum; suspensions; spectroscopy; flames

\section{Introduction}

Micron-sized metal powders, particularly aluminum, are common energetic additives to propellants and explosives. The mass fraction of metal in these energetic compositions can exceed $20 \%$, meaning that the metal burns as a dense suspension in the gaseous products of a hydrocarbon fuel matrix. Understanding the combustion behavior of metal particles in a dense suspension is central to the goal of predicting and tailoring the performance of metalized explosives and propellants. Metals are also increasingly being investigated for use as recyclable fuels [1, 2, 3], which would be burned in dense suspensions to achieve high power densities and ensure flame stability.

The combustion physics of a dense suspension of micron-sized particles can differ considerably from the combustion of large, individual, isolated particles, which have been the dominant subject of experimental work in metal combustion [4]. While large particles burn primarily in a diffusion-controlled regime of combustion, combustion of micron- and submicron-sized particles can be limited by heterogeneous kinetics or they can burn in a transitional diffusion-to-kinetic regime [5]. The combustion characteristics measured from single particle studies, such as combustion time and combustion regime, are often extrapolated to particles in the dense suspensions encountered in the majority of practical fuel systems. However, these characteristics are also functions of ambient temperature and oxidizer concentration and, therefore, will depend on the fuel particle concentration [6, 7]. The extrapolation of characteristic combustion data from single-particle studies to dense metal suspensions, where temperatures can exceed 
$3000 \mathrm{~K}$, will, in general, result in erroneous predictions. Thus, it is imperative to have experimental techniques to determine combustion characteristics of bulk reacting suspensions.

In our previous work, stabilized Bunsen-type flames were used to study combustion characteristics of aluminum and iron suspensions in oxygen and in the products of hydrocarbon flames [8, 9, 10, 11]. Other studies have used freely-propagating flames in tubes [12] or, more recently, transparent latex balloons [13], to study flame propagation speeds, laminar burning velocities, and the formation of instabilities. The burning velocities measured in these studies permit a more realistic estimation of the characteristic combustion time of the suspension compared to experiments with individual particles.

However, the determination of key combustion characteristics in suspensions of metal particles is difficult due to the inability to isolate and observe individual particles in the cloud. Optical diagnostic techniques to extract these parameters must be tailored to the specific nature of metal suspension combustion which can be optically dense, contain multiple temperatures, have intense luminosity, and cause strong multiple scattering. These phenomena are not often encountered in hydrocarbon combustion systems and largely prevent the use of "off-the-shelf" approaches to the study of combustion in suspensions.

Tailoring standard diagnostic techniques to metalized suspensions is often not straight forward. For example, recent studies show that coherent anti-Stokes Raman scattering (CARS) for gas temperature measurements in heavily metalized propellant flames may only be feasible with the use of femtosecond and picosecond laser pulses [14]. Using a technique like planar laser induced florescence (PLIF) to study the combustion of individual burning aluminum particles [15] is not realistic for quantitative measurements in suspensions due to the high particle number density. However, PLIF may still be useful as an imaging technique in these multi-phase environments [16].

Emission spectroscopy was previously applied to aluminum Bunsen flames to compare temperatures from gaseous $\mathrm{AlO}$ band spectra and condensed-phase continuum emitters, in an attempt to diagnose the particle combustion regime in the suspension and to compare temperatures to equilibrium calculations [17]. Line-of-sight integrated emission measurements from the cylindrically symmetric Bunsen flame cones required the use of a reverse Abel transform to reconstruct the radial profiles of spectral emission intensity to determine the thermal structure of the flame and the width of the combustion zone. The high optical thickness of both the condensed phase spectra and the AlO molecular bands, however, rendered the Abel reconstruction performed in [17] unreliable. The experiment was further complicated by the presence of strong multiple scattering of light by aluminum and aluminum oxide particles, which contaminated local emission spectra with the radiation from different regions of the flame. The accuracy of temperature measurements using continuum and molecular $\mathrm{AlO}$ spectra was also found to be insufficient to resolve the temperature difference between diffusively burning particles and the bulk flame temperature in the fuel-rich flames [17]. Due to these difficulties, the study was not able to draw definite conclusions about the regime of particle combustion in the suspension.

In the present study, a counterflow dust burner is used to create a flat aluminum dust flame with a one dimensional flame geometry that eliminates the need for spatial reconstruction using the Abel transform. Using the flat flame, an emission diagnostic technique that permits the direct qualitative determination of the regime of aluminum particle combustion in dense suspensions is demonstrated. The technique is based on the spectroscopic detection of selfreversal of the atomic aluminum emission lines that occurs due to inhomogeneous metal vapor temperatures near the micro-diffusion flames that surround the aluminum particles and in the bulk gas of the suspension. The diagnostic technique is also used to measure the combustion zone thickness. The length of the region where the lines are selfreversed serves as a measurement of the reaction zone and largely avoids the difficulties of interpreting the emission spectra in the presence of multiple scattering.

These measurements are compared to those obtained using a complementary diagnostic technique of spatially resolved absorption spectroscopy to detect the aluminum resonant lines. Using a broadband laser as a spectral source, the effects of multiple scattering are greatly reduced, and the length of the region where aluminum is detected serves as a measurement of the reaction zone. Combined with a particle image velocimetry (PIV) technique for estimating the particle residence time in the combustion zone and temperatures measured from $\mathrm{AlO}$ molecular spectra, the suite of diagnostics permits the characteristic combustion time of a dense aluminum suspension to be estimated. 


\section{Experimental methods}

\subsection{Counterflow burner and aluminum/gas mixtures}

The spectroscopic study is performed on flat aluminum dust flames stabilized using a counterflow dust burner (see Fig. 1a). The design of the apparatus is described in [9], where it was used to measure burning velocities in aluminum clouds using a PIV technique. For this study, the apparatus is modified to increase the flame planarity. The coaxial flow nozzle on the opposing jet in [9] is removed in order to eliminate recirculation of the combustion products on the flame periphery because it interferes with concentration and spectral measurements. The air in the coaxial flow around the bottom nozzle is also replaced with inert nitrogen to prevent formation of the diffusion flame often enveloping flat flames at fuel-rich conditions.

(a) flame image from DSLR

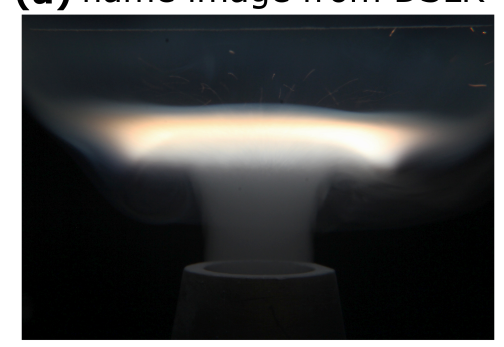

(b)

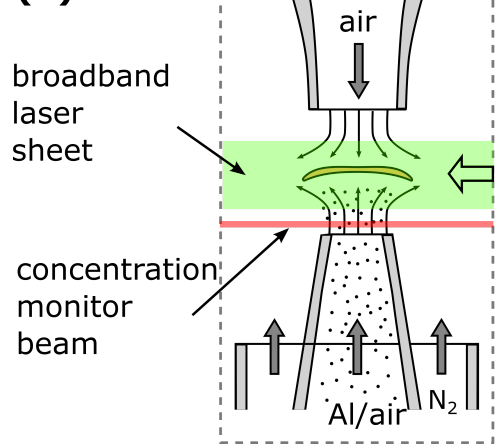

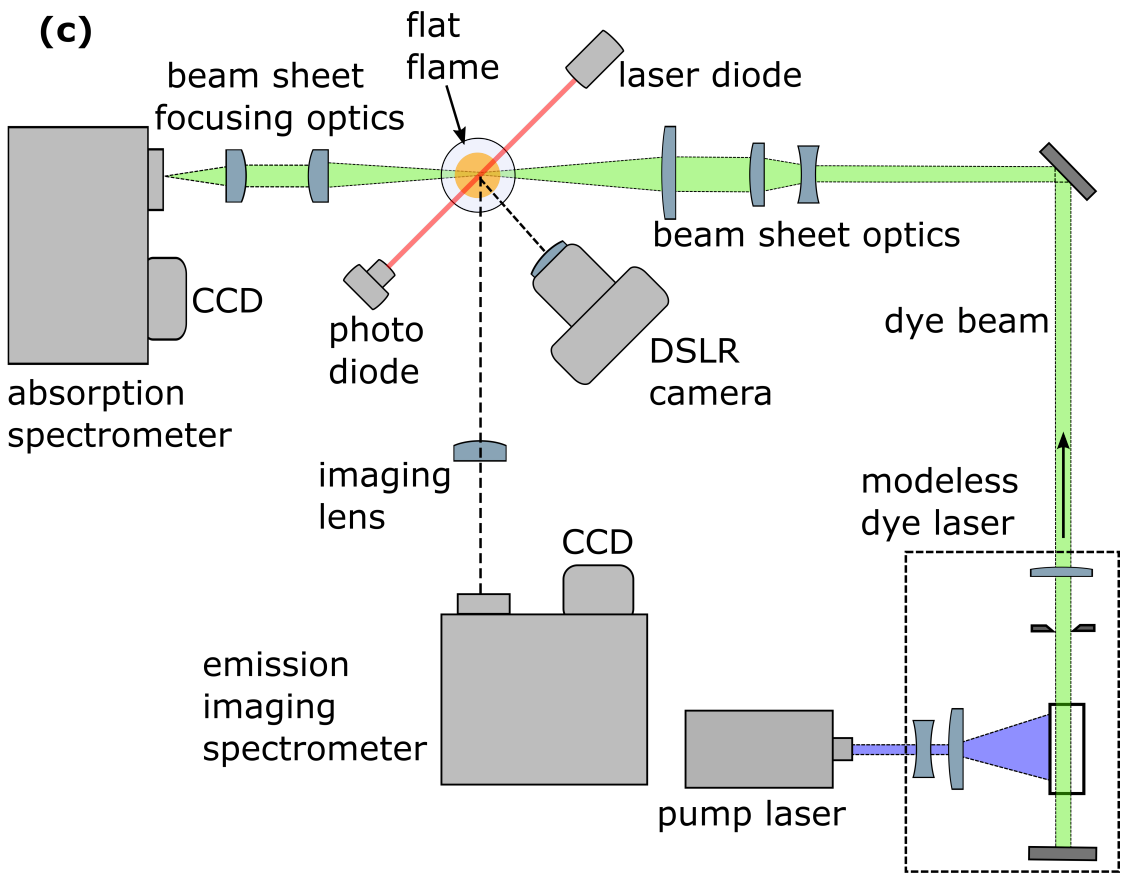

Fig. 1: a) Photograph of a flat flame in an aluminum-air suspension, b) schematic of the counterflow flame burner and side view of the laser sheet and dust concentration laser probe and c) top-view schematic of the imaging-emission and laser-absorption spectroscopy setups including simultaneous imaging and concentration measurement.

Concentration of the particles is monitored by a laser attenuation probe described in previous work [18]. In the present study, the laser beam probe passes just above the exit of the bottom nozzle as shown in Fig. 1b. An attempt was made to keep the concentration of aluminum in the suspension stable at around $300 \mathrm{~g} / \mathrm{m}^{3}$ for all measurements. Considering the accuracy of the dust concentration measurements, the actual concentration varies between 270 and $330 \mathrm{~g} / \mathrm{m}^{3}$.

The combustion in the aluminum suspension is studied in two gaseous environments: air and a stoichiometric methane-air mixture. The gas flow rates are monitored using factory calibrated electronic flow meters and rotameters. The oxygen concentration in aluminum-methane-air mixtures is monitored by an in-situ oxygen analyzer (Oxigraf) to determine the equivalence ratio.

\subsection{Aluminum powder}

Unlike our previous studies of stationary aluminum dust flames that used Ampal 637 aluminum (Ampal, Inc.) with nodular shaped particles with a size around $6 \mu \mathrm{m}[8,9,11]$, the present study employs $\mathrm{H}-2$ aluminum powder produced by Valimet Inc. (Stockton, CA) with spherical particles and a narrow particle size distribution. The particle size distribution in the H-2 powder from laser diffraction measurements is provided by Valimet and shown in Fig. 2 . The arithmetic mean diameter $\left(d_{10}\right)$ in the $\mathrm{H}-2$ aluminum powder is reported to be around $4.2 \mu \mathrm{m}$. 


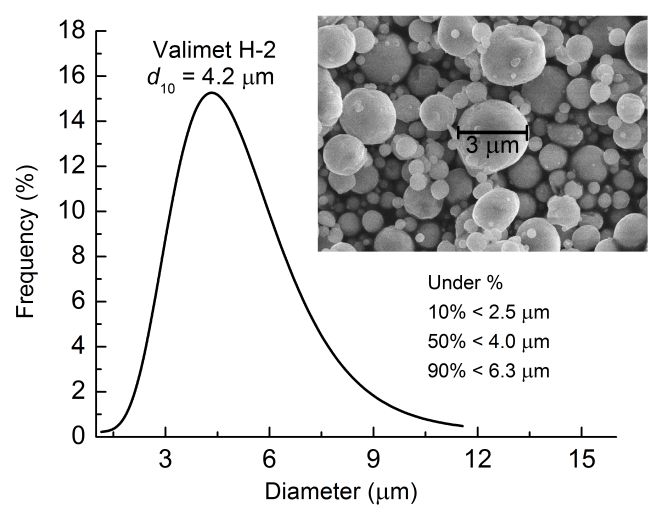

Fig. 2: Particle size distribution and SEM image of Valimet H-2 Aluminum. The particle sizing results are provided by the manufacturer.

\subsection{Emission imaging spectroscopy setup}

The general schematic of the imaging spectroscopy setup is shown in Fig. 1c. The setup consists of a $250 \mathrm{~mm}$ focal length spherical lens that images the flame onto the entrance slit of an imaging spectrometer with a focal length of $0.3 \mathrm{~m}$ equipped with a CCD array. Due to the internally recessed focal plane of the instrument, the CCD array detector is coupled to the spectrometer by a de-magnifying image relay consisting of two imaging lenses $(50 \mathrm{~mm}$ Nikkor $f / 1.4$ and $35 \mathrm{~mm}$ Nikkor $f / 2$ ). The detector is a CCD camera with a $4032(\mathrm{w}) \times 2688$ (h) pixel chip (Starlight Xpress SXVR H35) and $9 \mu \mathrm{m}$ square pixels. Only a $2006 \times 1336$ pixel segment of the CCD array is used as it affords faster image readout.

The spectrometer is installed with a $3600 \mathrm{gr} / \mathrm{mm}$ UV holographic grating. The spectrometer entrance slit width is set to $50 \mu \mathrm{m}$ permitting an overall spectral dispersion of $0.004 \mathrm{~nm} /$ pixel covering a spectral bandwidth of about $8 \mathrm{~nm}$ with an instrument resolution of about $0.03 \mathrm{~nm}$. For the measurements of the $\mathrm{AlO}$ molecular band spectrum, the holographic grating was replaced by a $1200 \mathrm{gr} / \mathrm{mm}$ ruled grating. This permits a spectral range of about $40 \mathrm{~nm}$ with an overall spectral dispersion of about $0.02 \mathrm{~nm} / \mathrm{pixel}$ and an instrument resolution of about $0.16 \mathrm{~nm}$.

\subsection{Absorption spectroscopy setup}

In order to perform absorption spectroscopy of the atomic aluminum vapor, a Q-switched Nd:YAG laser at the $3^{\text {rd }}$ harmonic (Quantel Ultra) is used to pump a modeless dye laser to produce a beam with broadband spectral output as outlined in previous publications [19, 20]. The dye used is a mixture of Exalite 398 and Exalite 389 (Exciton) at concentrations of $0.36 \mathrm{~g} / \mathrm{l}$ and $0.17 \mathrm{~g} / \mathrm{l}$, respectively, dissolved in reagent grade acetone. The pulse energy used in the present experiments is measured to be less than $0.5 \mathrm{~mJ}$ per pulse with a broadband spectral output of nearly $8 \mathrm{~nm}$ centered near $395 \mathrm{~nm}$. The broadband dye cell output covers both of the Al I doublet $\left({ }^{2} \mathrm{~S}_{1 / 2} \rightarrow{ }^{2} \mathrm{P}_{1 / 2,3 / 2}^{\mathrm{o}}\right)$ emission lines at 394.4 and $396.15 \mathrm{~nm}$.

The beam is expanded (focal length $f=-25 \mathrm{~mm}$ ), collimated $(f=50 \mathrm{~mm}$ ), and then focused into a narrow "sheet" using a cylindrical lens $(f=300 \mathrm{~mm})$. The sheet is passed through the flame and is focused several inches past the center of the flame in order to reduce the light collected from the flame emitters. The laser sheet is refocused, using a cylindrical lens, onto the slit of a customized spectrometer with a $0.45 \mathrm{~m}$ focal length installed with an $1200 \mathrm{gr} / \mathrm{mm}$ grating. The spectrometer is coupled to a CCD array by a de-magnifying image relay constructed from aspheric lens $(f=50 \mathrm{~mm})$ and a UV imaging lens $(f=25 \mathrm{~mm})$. The CCD is an EM-CCD (Rolera EM-C2) camera with a sensor size of $1004 \times 1002$ pixels and a pixel size of about $8 \mu \mathrm{m}$. This permits a dispersion of $0.02 \mathrm{~nm} / \mathrm{px}$ and an approximate instrument resolution of $0.12 \mathrm{~nm}$ covering a bandwidth of about $16 \mathrm{~nm}$. The camera trigger is synchronized to the laser pulse and the integration time of the camera is minimized to gate the laser pulse signal and minimize collection of the flame emission.

\subsection{Particle image velocimetry (PIV) setup}

A particle image velocimetry technique is used to determine the velocity of the aluminum particles as they enter the flame preheat zone. The details of the PIV technique and the specific difficulties for dust flames are discussed in a 
previous publication [9]. The beam from a $532 \mathrm{~nm}$ wavelength, $5 \mathrm{~W}$ continuous-wave laser (Dragon Lasers) is formed into a narrow light sheet using the same set of optical elements as in the laser absorption setup. The illuminated particles in the sheet are filmed at a frame rate of approximately 15000 fps by a Photron SA- 5 camera through a macro lens with a $532 \pm 2 \mathrm{~nm}$ narrow bandpass filter (Andover).

\section{General features of the emission and absorption spectra}

As practically no intrusive probes can be used for measurements inside flames of particulate suspensions, only optical methods, and specifically optical spectroscopy, can provide both qualitative and quantitative information on the flame structure. The present paper focuses primarily on the qualitative analysis of the spatially resolved aluminum atomic spectra that permits determination of the particle combustion regime in the flame and the estimation of the characteristic reaction time of suspension. Analysis of the other flame spectral characteristics is left to future work.

This study also highlights some of the specific difficulties encountered in optical diagnostics of flames in metal fuel suspensions, such as multiple light scattering by the condensed-phase media and large optical flame thickness. These effects, rarely encountered in gas-phase combustion, can easily be overlooked when applying similar spectroscopic methods to combustion of condensed-phase fuels.

\subsection{Aluminum atomic line emission spectra}

A sample emission spectrogram of the two strong atomic Al I lines at $394.4 \mathrm{~nm}$ and $396.15 \mathrm{~nm}$ emitted from the aluminum-air flame is shown in Fig. 3(left). The spectral intensity profiles at three flame locations are shown in Fig. 3(right). Two weak atomic lines observed near $393 \mathrm{~nm}$ are identified on the spectrogram as neutral Fe lines that are present due to iron impurities in the aluminum powder.
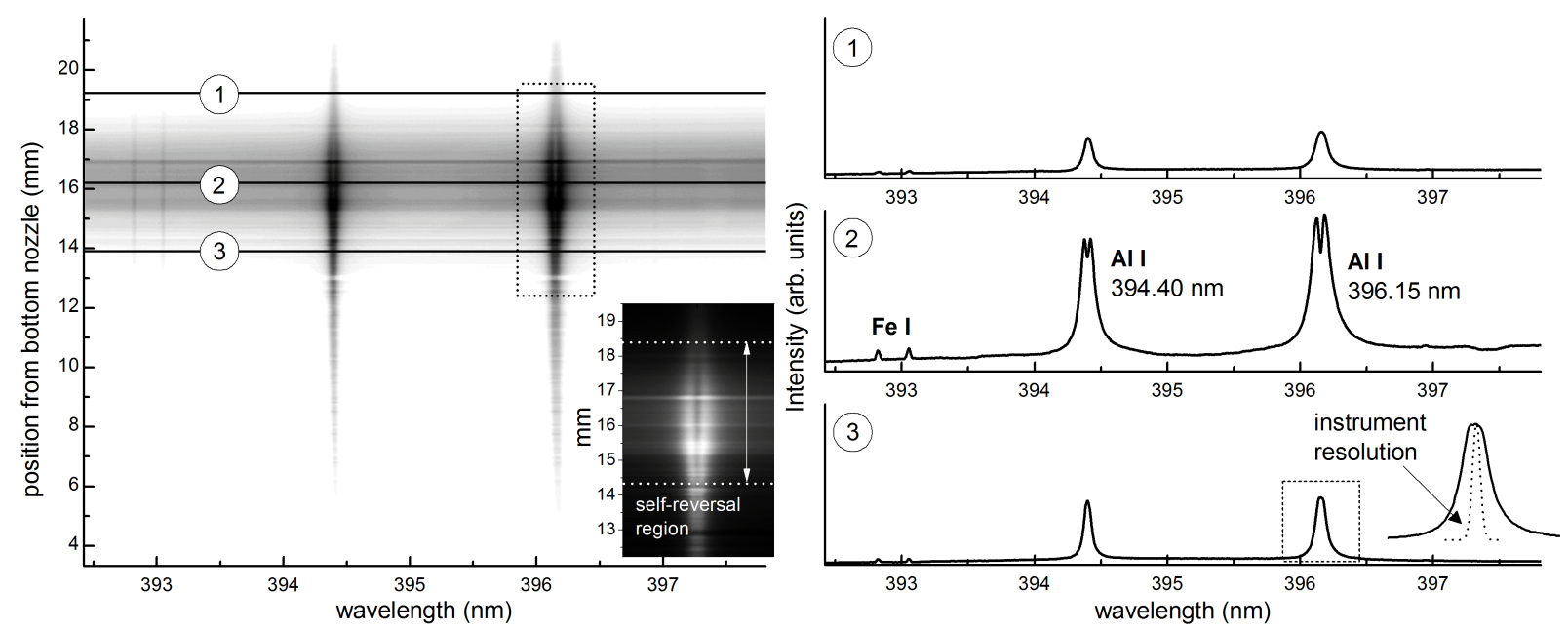

Fig. 3: (Left) Greyscale-inverted spectrogram of the aluminum atomic lines from aluminum-air flame with a (non-inverted) blown-up region of the self-reversal region in the inset. (Right) Intensity profiles of the spectrum taken at three different locations: (1) post-flame zone, (2) reaction zone, (3) pre-heat zone. The comparison of the emission line thickness to the instrument resolution function is also shown.

The $\mathrm{Al}$ atomic lines in the flame reaction zone appear to be strongly broadened. The observed broadening beyond the instrument resolution considerably exceeds the expected linewidth from Doppler-, collisional-, etc., broadening mechanisms and can only be attributed to strong self-absorption. The broadening effect of self-absorption is synonymous with large optical thickness and indicates high concentrations of the emitting/absorbing aluminum vapor species. In addition to the line broadening by self-absorption, the Al lines also demonstrate self-reversal in the flame reaction zone (see zone 2 in Fig. 3). The phenomenon of line self-reversal can occur only in the presence of a temperature gradient of the emitting/absorbing media along the line of sight [21], and is often observed in spectroscopic studies of plasmas [22] and widely used in astrophysics to infer the structure of stellar atmospheres [23].

Self-reversal is often observed in multi-temperature media due to the dependence of the atomic line broadening on temperature. The intensity profile of a resonance line, in both emission and absorption, from the hot atomic vapor 
will have a wider half-width than the colder regions of atomic vapor. The difference in line half-widths as well as emission intensity at different temperatures causes the observed atomic line on a spectrograph to be more strongly absorbed, by the colder atoms, in the center of the line than on the wings. The resulting profile of the emission line on a spectrometer is "caved-in" at the center, as shown for zone 2 of Fig. 3. The observation of line self-reversal, in general, requires not only temperature gradients in the emitting/absorbing media but will also only be apparent at sufficiently high optical depths with sufficiently high resolution of the spectral instrument. The conditions for the observation of line self-reversal have several other nuances which are outlined and discussed in [21].

In stark contrast to the results for the aluminum-air flame shown in Fig. 3, the results for aluminum-methane-air flames, shown in Fig. 4, indicate the absence of self-reversal of the aluminum lines in any region of the flame. The aluminum lines are also much thinner than in aluminum-air flames, with the line half-width nearly the same as that of the instrument function.
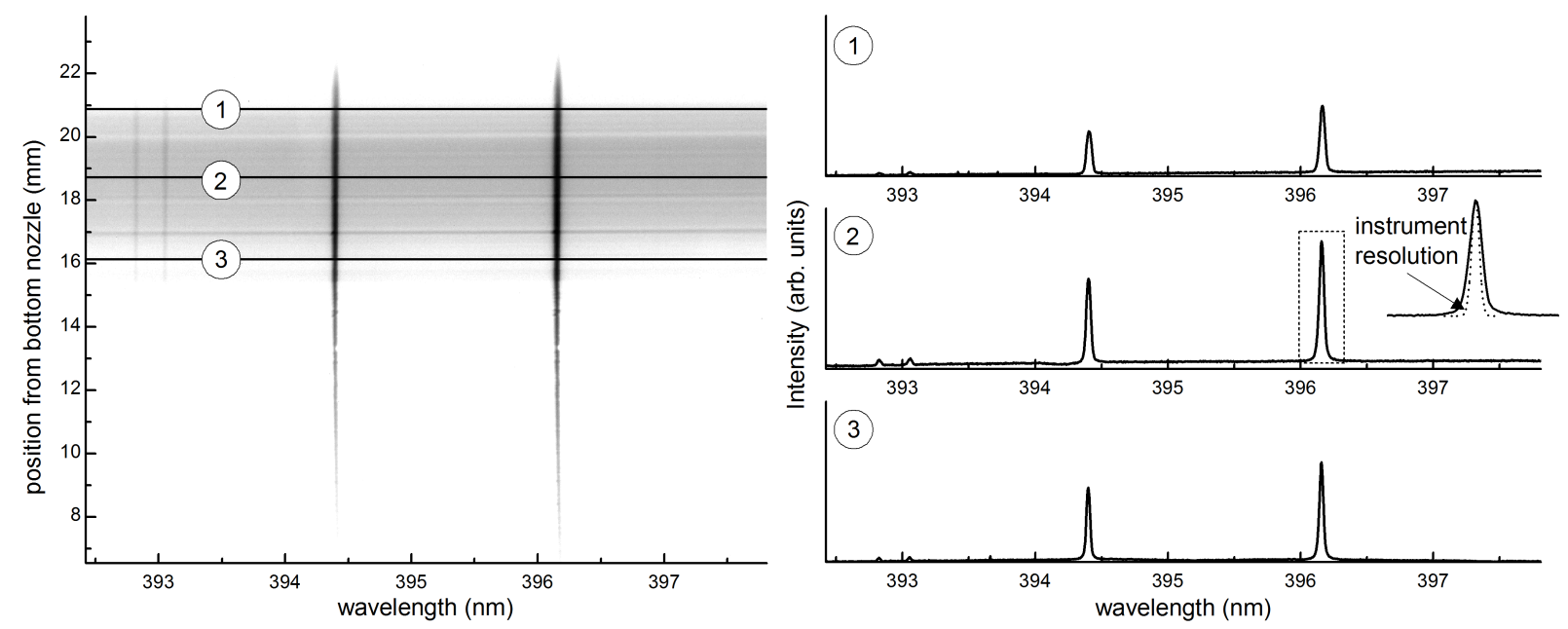

Fig. 4: (Left) Greyscale-inverted spectrogram of the aluminum atomic lines from the aluminum-methane-air flame showing no self-reversal. (Right) Intensity profiles of the spectrum taken at three different locations: (1) post-flame zone, (2) reaction zone, (3) pre-heat zone. The comparison of the emission line thickness to the instrument resolution function is also shown.

Taking into account the similar flame geometries and light path lengths through the flames in both mixtures, the observed differences in line-widths suggest a much higher optical thickness of aluminum lines in aluminum-air suspensions compared to the aluminum-methane-air mixture which, in turn, is indicative of a much larger concentration of aluminum vapor in aluminum-air flames. The plausible explanation for why line self-reversal is, or is not, observed in aluminum-air and aluminum-methane-air flames is further discussed in the Discussion section.

\subsection{Aluminum atomic line absorption spectra}

A typical image of the absorption spectrogram through the flame is shown in Fig. 5 for both air and methane-air oxidizing mixtures. The spectral profile of the modeless dye laser exhibits a relatively strong noise level, as was observed in previous studies [19]. There are also variations in intensity along the spatial axis due to the non-uniform beam profile of the laser. Variable attenuation from particles in the medium and small imperfections in the spectrometer slit also create striations in the laser intensity along the spatial coordinate. Despite this non-uniformity, the laser sheet technique can still yield a relatively clear picture of the line-of-sight spatial profiles of $\mathrm{Al}$ absorption lines, permitting a reconstruction of spatial distribution of aluminum vapor concentration in the flame.

The emission spectrograms in Fig. 3 and Fig. 4 reveal long zones of aluminum vapor emission along the spatial coordinate compared to the absorption spectrograms in Fig. 5. The absorption measurements reveal a zone of aluminum vapor less than a couple of millimeters in length while the emission measurements indicate that aluminum vapor can apparently be observed well above and below the flame zone even near the cold, dispersion nozzle. The observed difference can be explained by the strong multiple scattering of light emitted from the combustion zone by both the unburned aluminum particles in the preheat zone and nano-sized oxides in the post-flame zone. In contrast to the emission measurement, the highly directional laser absorption measurements shown in Fig. 5 are not strongly 
affected by the scattered light and, thus, provides a more realistic measurement of the location of aluminum vapor in the flame.

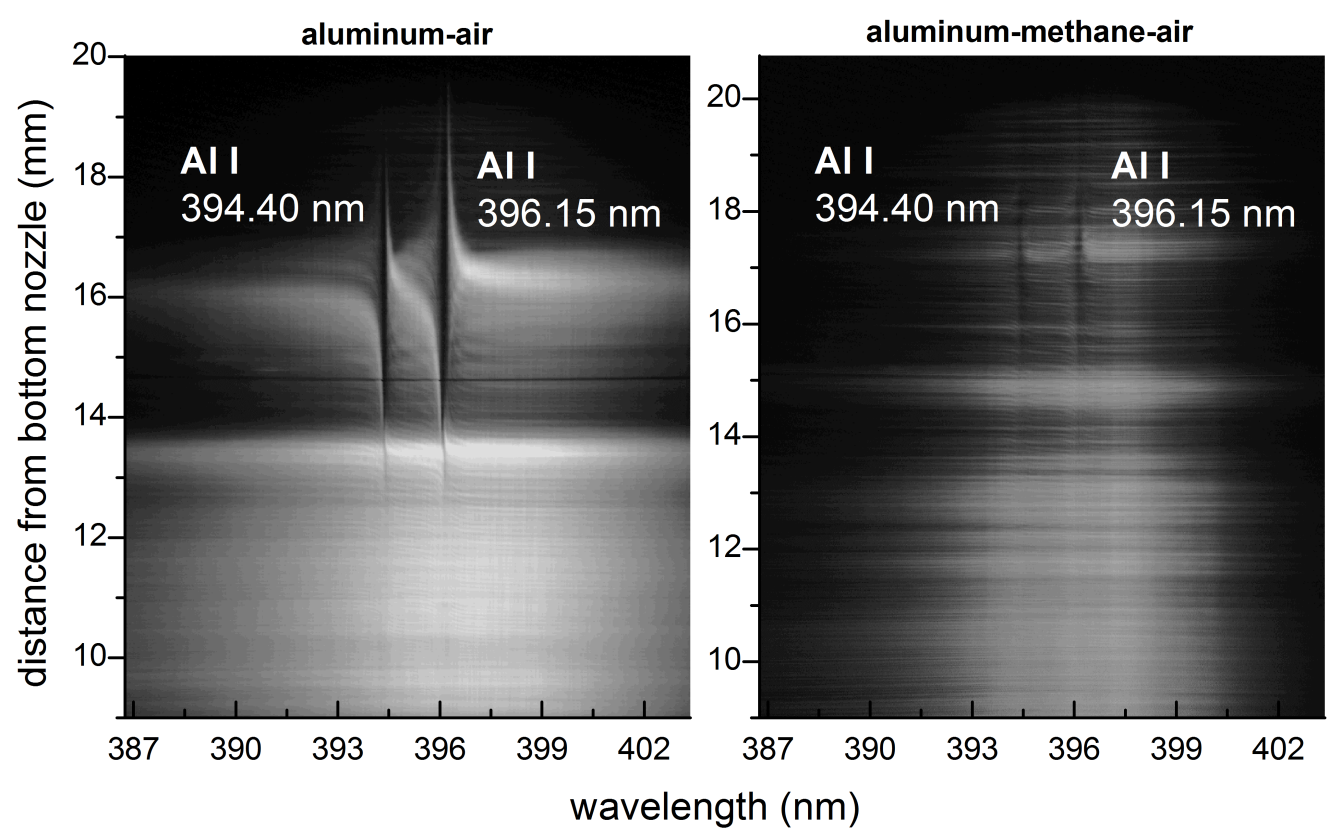

Fig. 5: Absorption spectrograms of the Al I atomic lines for aluminum-air and aluminum-methane-air flames.

The spatial variations of the laser beam intensity in Fig. 5 also reveal an interesting phenomenon showing that light at wavelengths near the resonance wavelength bends upward or downward along the spatial axis in the aluminum-air flame. This can also be observed, albeit to a lesser extent, in the aluminum-methane-air spectrogram.

The bending observed here is qualitatively similar to the effect observed in the combined spectroscopic and interferometric technique known as the Hook Method which exploits anomalous dispersion near the resonance lines to measure concentration of species [24]. In the present study, the observed light bending is also due to anomalous dispersion but without the interference fringes seen in the hook method. In these spectrograms, the combustion of the aluminum particles in the flame produces a strong spatial gradient of aluminum vapor. At high aluminum vapor concentrations, anomalous dispersion causes pronounced deviations in the refractive index of the medium at wavelengths near the aluminum resonance lines. The strong spatial concentration gradients, and corresponding gradients in refractive index, lead to a pronounced lensing effect where the flame essentially behaves as a gradient index lens for the laser light frequencies around resonance lines. The lensing effect stretches the spatial appearance of aluminum lines and makes the interpretation of the spatial intensity profiles of absorption spectra difficult.

Nevertheless, the degree of lensing that occurs and the width of the absorption line give direct qualitative indication of the amount of aluminum vapor in the reaction zone. It can be easily seen that the spectrogram for aluminum-air flames exhibits a stronger lensing effect, as well as much wider absorption lines, compared to aluminum-methane-air flames. This confirms the results from emission spectroscopy, where considerably higher concentrations of aluminum vapor are observed in aluminum-air flames in comparison to aluminum-methane-air flames.

\subsection{Molecular emission spectra of AlO}

The previous spectral study of the Bunsen aluminum dust flame attempted to use the $\mathrm{AlO}$ emission spectrum and the condensed phase continuum spectrum to derive spatial distributions of temperatures in the flame [17]. The present results, however, indicate that strong multiple scattering of the light in two-phase metal flames makes spatial interpretation of the emission spectra unreliable. At best, the emission measurements can only indicate the maximum temperature in the flame, since emission intensity is strongly weighted to higher temperatures [25].

The measurement of condensed-phase temperatures, by fitting the continuum spectrum to a Planck distribution, relies on the choice of an appropriate spectral emissivity function of the aluminum oxide emitters, which, in general, is a strong function of wavelength, temperature, and the optical depth [26, 27]. As shown in the literature, a 
small deviation in emissivity data can lead to large scattering in temperatures, often reaching several hundred degrees [28]. Due to these difficulties, we have opted to derive gas temperatures only from AlO molecular spectra in this study. As in previous work [17], the temperature is found by fitting a simulated spectral intensity distribution in the $\mathrm{AlO} \mathrm{B}-\mathrm{X} \Delta \nu=-1$ band sequence to the experimentally measured spectral intensity.

The $\mathrm{AlO} \mathrm{B}-\mathrm{X} \Delta \nu=-1$ band sequence emitted by the aluminum-air flame is shown in Fig. 6 . Similar to the $\mathrm{Al}$ atomic lines, the $\mathrm{AlO}$ molecular lines become optically thick in the flame zone (Fig. 6(right), zone 1). Away from the flame, the spectrum is optically thin, and, thus, fits reasonably well with a simulation based on optically thin approximations commonly used in literature [29]. In the optically thick zone, a temperature fitting of the AlO band sequence becomes much less accurate. The effect of optical thickness is to enhance the weak lines at the expense of the stronger features. This effect typically skews fits to higher temperature as shown in Fig. 6(right) (zone 1). Here the observed spectrum is compared to optically thin spectra simulated using the ExoMol line list for the AlO B-X transition simulated in the PGopher software [30, 31]. It appears that even when optical thickness is accounted for in the model, the relative line intensities become less sensitive to temperature leading to considerable uncertainty in measurements.
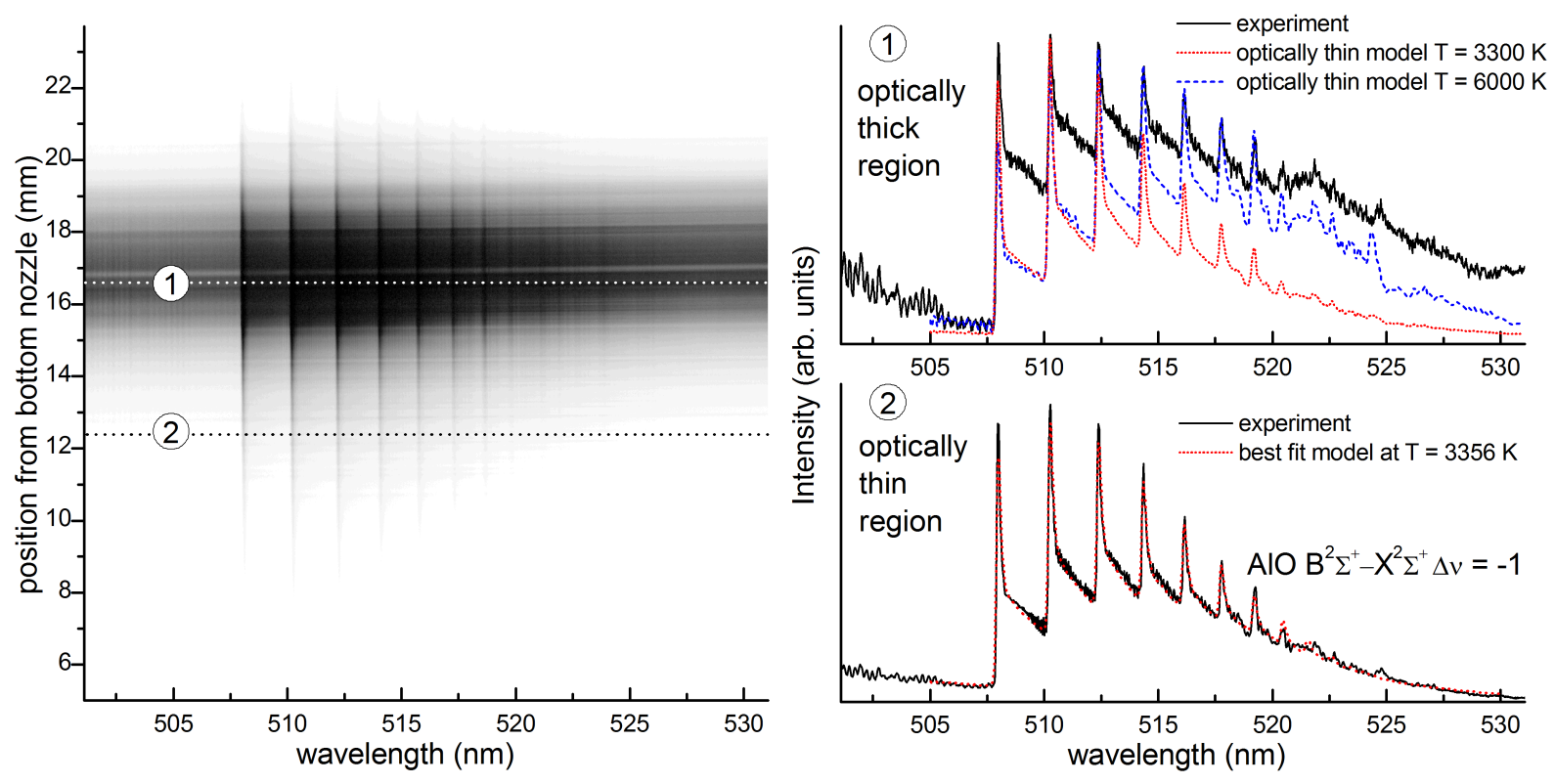

Fig. 6: (Left) inverted spectrogram of the AlO B $-\mathrm{X} \Delta \nu=-1$ band sequence across the flame. (Right) Spectra from two locations in the flame showing the optically thick and optically thin regions.

The light emission observed from the cold, pre-flame zone (below zone 3 on Fig. 3 and Fig. 4) is due to multiple scattering of light from the reaction zone in flame. Scattered light from the flame, in general, will have traveled a smaller distance through the reaction zone in comparison to the light from the flame traveling along the optical axis and is, consequently, an optically thinner version of the spectrum from the flame zone. Due to the strong bias of resonant emission to high temperatures, measurement using scattered light generally can only indicate the maximum temperature of $\mathrm{AlO}$ in the flame. In the present experiment, measurements of temperatures at different locations on the spectrogram outside of the combustion zone yield nearly the same temperatures regardless of the location, even in the cold zone upstream of the flame.

\subsection{Comparison of flame temperatures derived from AlO spectra with equilibrium calcula- tions}

The resulting temperatures derived from the optically thin AlO spectra are plotted in Fig. 7 together with adiabatic flame temperature, and equilibrium concentrations of aluminum vapor calculated with the NASA CEA code [32].

Equilibrium calculations predict close to $400 \mathrm{~K}$ temperature difference between aluminum-air and aluminummethane-air flames, while the actual measured temperature difference is considerably smaller. The average tempera- 
ture measured in aluminum-air flames is about $3370 \mathrm{~K}$ while the average temperature measured in aluminum-methaneair flames is found to be about $3170 \mathrm{~K}$, with an estimated measurement accuracy of about $\pm 150 \mathrm{~K}$ in both cases [29].

It needs to be noted that equilibrium calculations predict higher concentrations of aluminum vapor in aluminummethane-air mixtures in comparison to aluminum-air mixtures. The emission and absorption spectroscopy results show the exact opposite behavior. The possible explanation for the difference between the measurements and equilibrium calculations is discussed in the Discussion section.

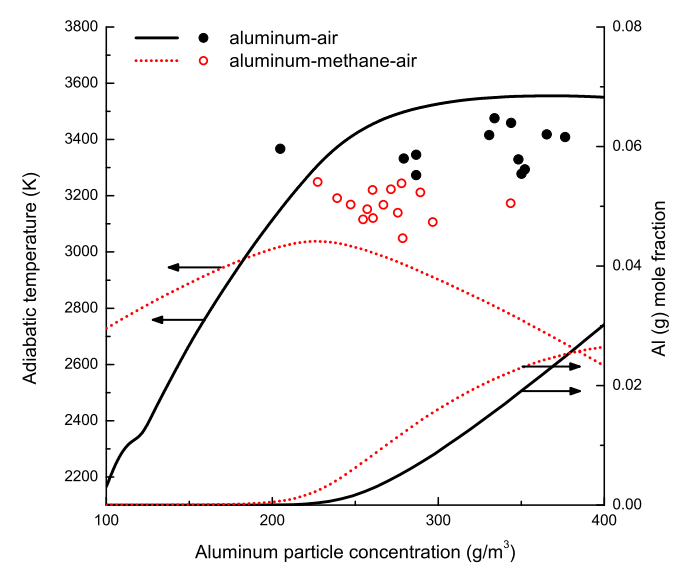

Fig. 7: Flame temperatures measured using AlO molecular spectra (• and o) in comparison to equilibrium calculations (lines) at different initial aluminum particle concentrations. The predicted mole fraction of gaseous aluminum vapor in equilibrium is also shown.

\section{Discussion}

\subsection{Al emission self-reversal and the regime of combustion in the flame}

In the majority of models in the literature [33], diffusion limited combustion of aluminum particles is similar to hydrocarbon droplet combustion during which aluminum vapor generated by the evaporating aluminum droplet reacts with oxygen in a thin gas-phase flame enveloping the droplet at the distance of several droplet radii. The temperature of such micro-diffusion flames, lifted from the particle surface, is close to the adiabatic temperature of the stoichiometric aluminum mixture with oxidizer. It can be several hundred degrees higher than the temperature of the evaporating aluminum droplet and the average temperature of the gas between the reacting particles.

The existence of diffusion micro-flames around the particles leads to local micro-gradients of aluminum vapor concentration and temperature within the suspension. The high reactivity of aluminum vapor with various oxidizers [34, 35], generally prevents the formation of a premixed aluminum-oxidizer mixture, such that the aluminum vapor can only exist in the bulk gas of a fuel-rich mixture once the oxidizer has been nearly consumed. For nearly stoichiometric mixtures, a small amount of aluminum vapor forms due to natural dissociation process of aluminum sub-oxides at high temperatures. Traversal of the light emitted by micro-diffusion flames through colder aluminum vapor in the space between the flame and the particle surface, and in the bulk gas, leads to the self-reversal ("caving") of aluminum lines. This self-reversal results from the different half-width of the Al lines at different temperatures, as illustrated in Fig. 8.

Self-reversal of aluminum atomic lines was also observed in our previous work on spectroscopy of aluminum Bunsen-type conical flames [17], where the lines were also often caved-in below the intensity of the continuous spectrum. This lineshape was believed to be caused by the macro-temperature gradients along the line-of-sight due to the cylindrical flame geometry, where light emitted from the flame front travels through cooling combustion products containing residual aluminum vapors due to the fuel-rich combustion. The use of the flat flame in the present work precludes the existence of macro-scale temperature gradients along the line-of-sight, suggesting that the observed strong self-reversal of aluminum lines in aluminum-air flames is the result of the micro-scale temperature gradients of the aluminum vapors inside the lifted micro-diffusion flames that surround burning aluminum droplets. It is interesting to note that the self-reversal of aluminum and magnesium atomic lines was first noticed during a spectroscopic study 


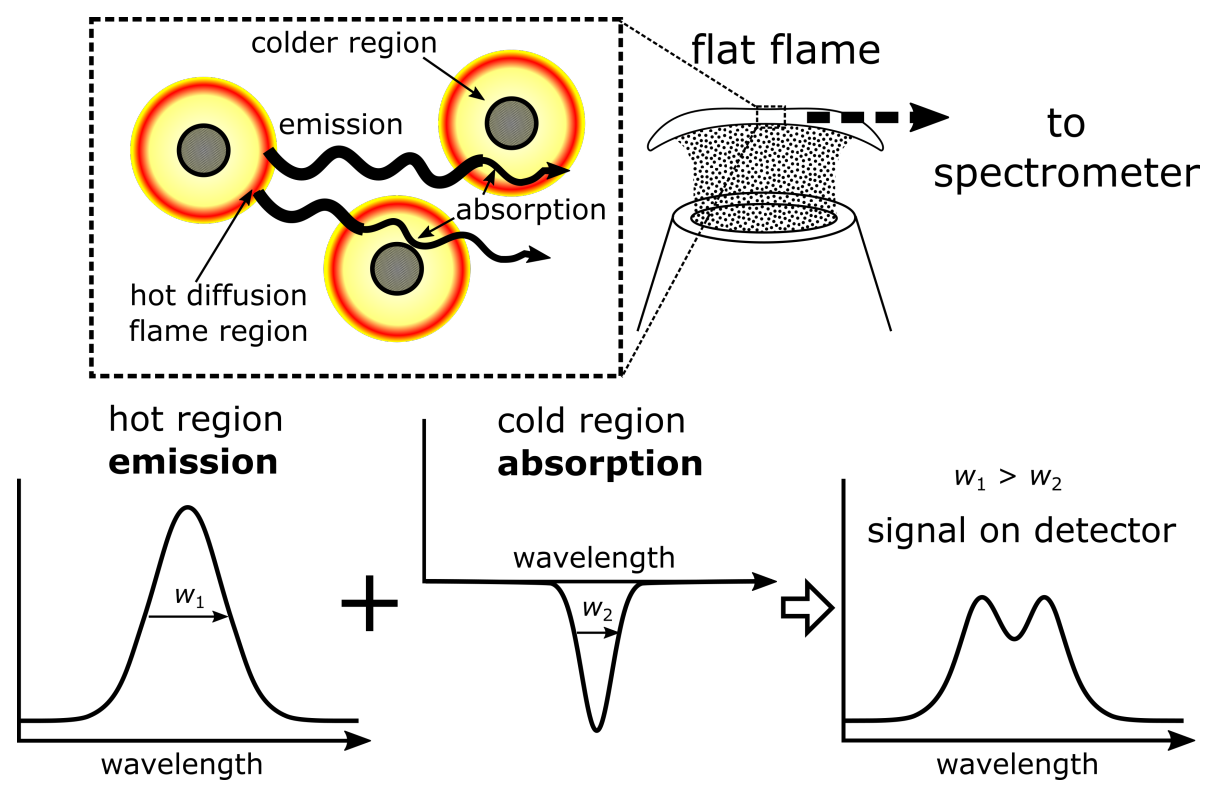

Fig. 8: (Top) schematic of the micro-scale emission and absorption of aluminum vapor from the flat flame that is integrated along the line of sight to the spectrometer. (Bottom) Schematic of self-reversal. Hot zone emission of linewidth $w_{1}$ is partially absorbed by cold zone with linewidth of $w_{2}$ where $w_{1}>w_{2}$.

of the combustion of burning magnesium ribbons and aluminum foils, which was also interpreted as evidence of a diffusion-dominated combustion regime due to the presence of multiple temperatures [36].

The diffusion regime of combustion causes high concentrations of aluminum vapors in the space between the aluminum droplet and the micro-diffusion flame, and these vapors are insulated by the flame from the oxidizers within the bulk gas. The observed large optical depths of Al emission and absorption lines indeed indicate very high aluminum vapor concentrations in aluminum-air flames and point to a diffusion-limited flame mode. In this regime of particle combustion, there is no macro-equilibrium state for a given flame cross section, and the temperature and integrated concentrations of gaseous aluminum in the flame can exceed the predicted equilibrium values of the bulk mixture. A similar conclusion was also made by in the study of laminar diffusion flames in aluminum suspensions, where the high optical depth of the atomic Al lines was also attributed to the existence of micro-diffusion flames [37].

In contrast to aluminum-air flames, no line self-reversal was observed in aluminum-methane-air flames. In addition, the atomic Al lines emitted by these flames are generally optically thin, with the line half-width on the order of the instrument resolution. The absence of the line self-reversal, and lower concentration of aluminum vapors indicated by the weak broadening of $\mathrm{Al}$ lines, suggest that, unlike in aluminum-air flames, lifted diffusion micro-flames do not form in aluminum-methane-air suspensions. This observation points to a heterogeneous, and most likely kinetically-limited, combustion mode of aluminum in the hydrocarbon combustion products. The disparity between the equilibrium calculations that predict similar concentrations of aluminum vapor in both mixtures, shown in Fig. 7, and the large difference in the spectroscopically measured concentrations is explained by the different modes of combustion in each mixture. A shift to kinetically dominated combustion in methane-air mixtures is consistent with the conclusions of our previous hybrid aluminum-methane Bunsen flame studies [8, 18]. While a detailed analysis as to why the change in combustion regime occurs is beyond the scope of the present work, it is not surprising taking into account the slower rates of heterogeneous aluminum reactions with $\mathrm{CO}_{2}, \mathrm{CO}$, and $\mathrm{H}_{2} \mathrm{O}$ [4], as well as higher rates of molecular heat transfer in the media containing water molecules and the hydrogen produced by the aluminum-water reaction.

\subsection{The width of the flame reaction zone}

The thickness of the combustion zone in the flame can be estimated from the spatially resolved spectrogram of aluminum vapor lines in both emission and absorption. As mentioned previously, the strong multiple scattering of the light by unreacted aluminum particles and nano-sized oxide products makes the determination of the location of the aluminum vapor from the intensity of the emitted light unreliable. This causes the luminous zone of the flame to be much larger than the reaction zone thickness. It is possible, however, to use a spatially resolved measurement of the 
self-reversed line, when it is present, as a method to estimate the flame thickness. The presence of self-reversal on the emission spectrogram is not as affected by multiple scattering since, in order for self-reversal to occur, light must traverse a significant path along the optical axis through the flame zone. Therefore, self-reversal is practically not present in the scattered light.

A self-reversed line appears as a double-peaked spectral feature with a local minimum at the central resonance wavelength. The ratio of the intensity at the local minimum at the line center to the peak intensity on either side of the line is a measure of the relative degree of self-reversal. When this ratio is unity, self-reversal is weak either due to low optical depth or small temperature gradients within the medium. When this ratio is close to zero, the self-reversal phenomenon is strong, indicating temperature gradients and typically high optical depths. The value of this spectral intensity ratio subtracted from unity measured along the spatial axis of the spectrogram is a peaked function with a half-width that serves as an estimate for the flame thickness. The average "self-reversal width" measured for the $396.15 \mathrm{~nm} \mathrm{Al} \mathrm{I} \mathrm{line} \mathrm{in} \mathrm{aluminum-air} \mathrm{mixtures} \mathrm{over} \mathrm{several} \mathrm{spectrograms} \mathrm{indicates} \mathrm{a} \mathrm{combustion} \mathrm{zone} \mathrm{thickness} \mathrm{on} \mathrm{the}$ order of $1.7 \pm 0.3 \mathrm{~mm}$. The reported error represents the standard deviation over the measurements.

The spatially resolved absorption profiles of $\mathrm{Al}$ lines in Fig. 5 are not as affected by multiple light scattering and, thus, can also be used to estimate the thickness of the combustion zone. They are, however, spatially distorted by the lensing effect from anomalous dispersion around the resonance line. Nonetheless, the theory of anomalous dispersion indicates that the index of refraction remains unperturbed at the line center. Therefore, the real unperturbed spatial length of the aluminum absorption line can be reconstructed by plotting the intensity of the aluminum absorption line in its center compared to the unperturbed laser continuum.

The intensity of absorption at the central line wavelength was measured in relation to the unabsorbed and unperturbed baseline laser spectrum intensity, taken far from the flame zone and away from wavelengths demonstrating the lensing effect. The width of the resulting spatial distribution of intensity in the atomic line center is used as an estimate of the combustion zone thickness. Using this method, a combustion zone thickness in aluminum-air flames, averaged from five spectrograms, is estimated to be about $2.3 \pm 0.5 \mathrm{~mm}$, i.e. about $25 \%$ larger than the estimate given by the method of the line self-reversal but not significantly different given the uncertainties in each measurement.

The estimated length of aluminum vapor in aluminum-methane-air flames of about $1.5 \pm 0.7 \mathrm{~mm}$ using this method may reflect only part of the combustion zone length where the particles burn in the intermediate diffusion-to-kinetic regime as discussed in recent works [6, 7]. Aluminum absorption lines could also be an indication of hot aluminumvapor in the equilibrated bulk gas predicted by thermodynamics (see Fig. 7). This observation would suggest that the length of aluminum vapor lines reflects cooling of the products and has no correlation with the particle combustion zone.

\subsection{Estimation of the suspension combustion time}

The characteristic reaction time of the suspension can be estimated by dividing the length of the flame reaction zone by the average velocity of the burning particles traversing it. The velocity at which the particles enter the flame is determined from the axial velocity profiles of the particles measured by the PIV technique, where aluminum particles also play the role of the flow tracers. The particles slow down as they approach the stagnation surface and their velocity reaches a minimum as they enter the preheat zone of the flame [38]. After this point, the gas expands due to heating and particles accelerate (see [9]). Due to the very small velocity slip estimated between the H-2 aluminum particles and the gas, the measured particle minimum velocity practically coincides with the velocity of gas and, therefore, is the burning velocity of the flame. Indeed, the measured values of the minimum particle speed in stoichiometric methane-air flames using low loadings of H-2 aluminum particles exhibited values very close to the methane-air burning velocities reported in the literature [39], indicating that the slip between the H-2 particles and the gas is small.

The PIV technique cannot track the reacting particles in the flame as they are rapidly obscured by nano-sized oxide combustion products. As a result, the particle velocity at the end of the combustion zone was estimated by multiplying the entrance speed by the gas expansion coefficient calculated by a ratio of the flame temperature and room temperature. The average minimum particle speed and, therefore, burning velocity in aluminum-air flames is measured to be about $48 \pm 6 \mathrm{~cm} / \mathrm{s}$. The measured burning velocity for $\mathrm{H}-2$ Valimet powder is considerably higher than the measured burning velocities of $6 \mu \mathrm{m}$ Ampal-637 powder of about $35 \mathrm{~cm} / \mathrm{s}$ [9]. This observation corresponds to the expected, inversely proportional, dependence of the flame burning velocity on particle size [6]. The particles are assumed to accelerate constantly to their maximum velocity set by the expansion ratio, which is determined from the average flame temperatures measured by the $\mathrm{AlO}$ spectra (Fig. 7). The average particle velocity is determined to be $\approx 295 \mathrm{~cm} / \mathrm{s}$ for flames in aluminum-air suspensions. This gives an estimate for the characteristic reaction time for 
a nearly stoichiometric aluminum-air suspension of about $0.6 \mathrm{~ms}$ using the flame thickness determined by the line self-reversal method and about $0.8 \mathrm{~ms}$ using the flame thickness profiles found by spectral absorption.

As mentioned previously, the length of aluminum lines measured in aluminum-methane-air flames are not directly related to the flame combustion zone and, therefore, some other markers that can indicate heterogeneous and kinetically-controlled aluminum combustion will need to be developed in future studies.

It should also be noted that the methods of flame thickness measurement in the aluminum-air flames are biased toward the combustion of larger particles which would be expected to produce more aluminum vapor from the microdiffusion flames and to have comparatively longer burning times. The zone of the majority of heat release may be much smaller in comparison to the zone of aluminum vapor and, thus, it is likely that the flame thickness and combustion time are shorter than currently estimated.

\section{Conclusion}

Flat flames in aluminum-air and aluminum-methane-air suspensions, stabilized using a counterflow burner, are investigated using imaging emission spectroscopy, laser-absorption spectroscopy, and PIV. It is demonstrated that the combination of these optical techniques can be used to extract the combustion characteristics of particles in dense suspensions where isolating and observing individual particles is nearly impossible.

The emission diagnostics show that aluminum-air flames exhibit strong self-reversal of atomic aluminum resonance lines in the combustion zone as well as high optical depths in both emission and absorption spectra. Both of these observations are attributed to aluminum particles burning in a diffusion-controlled combustion regime with the formation of lifted micro-diffusion flames around the particles. In contrast, the aluminum resonance lines in the aluminum-methane-air flames show no self-reversal and are more optically thin in both emission and absorption spectra, indicating more heterogeneous, and likely kinetically-controlled, aluminum combustion. The combination of combustion zone thickness measurements from spatially resolved emission and absorption spectra, the flame temperatures derived from $\mathrm{AlO}$ molecular spectra, and the particle velocities measured by PIV provide the necessary information to estimate the combustion time of the particle suspension. The estimated characteristic combustion time of the aluminum-air suspension is found to be in the range of 0.6 to $0.8 \mathrm{~ms}$.

The diagnostic approach outlined in this paper provides a methodology for studying particle combustion at more realistic particle loadings encountered in practical fuel systems. These diagnostics are important for understanding burning behavior and the contribution of particle energy release to the flow field in dense suspensions.

\section{Acknowledgments}

Support for this work was provided by the Defense Threat Reduction Agency under contract HDTRA1-11- 1-0014 and a McGill Engineering Doctoral Award. Additional funding was provided by the Panda Faculty Scholarship in Sustainable Engineering \& Design and a William Dawson Scholarship.

\section{References}

[1] J. Bergthorson, S. Goroshin, M. Soo, P. Julien, J. Palecka, D. Frost, D. Jarvis, Direct combustion of recyclable metal fuels for zero-carbon heat and power, Appl. Energy 160 (2015) 368-382.

[2] M. Schiemann, J. Bergthorson, P. Fischer, V. Scherer, D. Taroata, G. Schmid, A review on lithium combustion, Appl. Energy 162 (2016) 948-965.

[3] J.M. Bergthorson, Y. Yavor, J. Palecka, W. Georges, M. Soo, J. Vickery, S. Goroshin, D.L. Frost, A.J. Higgins, Metal-water combustion for clean propulsion and power generation, Appl. Energy 186 (2017) 13-27.

[4] M.W. Beckstead, B.R. Newbold, A summary of aluminum combustion, Proc. 50th JANNAF Propuls. Meet. 705 (2001) 201-220.

[5] T. Bazyn, H. Krier, N. Glumac, Evidence for the transition from the diffusion-limit in aluminum particle combustion, Proc. Combust. Inst. 31 (2007) 2021-2028. 
[6] M.J. Soo, K. Kumashiro, S. Goroshin, D.L. Frost, J.M. Bergthorson, Thermal structure of flames in non-volatile fuel suspensions, Proc. Combust. Inst. 36 (2017) 2351-2358.

[7] M. Soo, S. Goroshin, J.M. Bergthorson, D.L. Frost, Reaction of a particle suspension in a rapidly-heated oxidizing gas, Propellants, Explos. Pyrotech. 40 (2015) 604-612.

[8] M. Soo, P. Julien, S. Goroshin, J.M. Bergthorson, D.L. Frost, Stabilized flames in hybrid aluminum-methane-air mixtures, Proc. Combust. Inst. 34 (2013) 2213-2220.

[9] P. Julien, S. Whiteley, M. Soo, S. Goroshin, D.L. Frost, J.M. Bergthorson, Flame speed measurements in aluminum suspensions using a counterflow burner, Proc. Combust. Inst. 36 (2017) 2291-2298.

[10] P. Julien, S. Whiteley, S. Goroshin, M.J. Soo, D.L. Frost, J.M. Bergthorson, Flame structure and particlecombustion regimes in premixed methane-iron-air suspensions, Proc. Combust. Inst. 35 (2015) 2431-2438.

[11] S. Goroshin, I. Fomenko, J.H.S. Lee, Burning velocities in fuel-rich aluminum dust clouds, Proc. Combust. Inst. 26 (1996) 1961-1967.

[12] J. Palecka, P. Julien, S. Goroshin, J.M. Bergthorson, D.L. Frost, A.J. Higgins, Quenching distance of flames in hybrid methane-aluminum mixtures, Proc. Combust. Inst. 35 (2015) 2463-2470.

[13] P. Julien, J. Vickery, S. Goroshin, D.L. Frost, J.M. Bergthorson, Freely-propagating flames in aluminum dust clouds, Combust. Flame 162 (2015) 4241-4253.

[14] S.P. Kearney, D.R. Guildenbecher, Temperature measurements in metalized propellant combustion using hybrid fs/ps coherent anti-Stokes Raman scattering, Appl. Opt. 55 (2016) 4958.

[15] P. Bucher, R. Yetter, F. Dryer, T. Parr, D. Hanson-Parr, E. Viceni, Flames structure measurement of single, isolated aluminum particles burning in air, Symp. (Int.) Combust. 26 (1996) 1899-1908.

[16] T.D. Hedman, K.Y. Cho, M.A. Pfeil, A. Satija, H.C. Mongia, L.J. Groven, R.P. Lucht, S.F. Son, High speed OH PLIF applied to multiphase combustion (Review), Combust. Explos. Shock Waves 52 (2016) 1-13.

[17] S. Goroshin, J. Mamen, A. Higgins, T. Bazyn, N. Glumac, H. Krier, Emission spectroscopy of flame fronts in aluminum suspensions, Proc. Combust. Inst. 31 (2007) 2011-2019.

[18] P. Julien, M. Soo, S. Goroshin, D.L. Frost, J.M. Bergthorson, N. Glumac, F. Zhang, Combustion of Aluminum Suspensions in Hydrocarbon Flame Products, J. Propuls. Power 30 (2013) 1-8.

[19] N. Glumac, Absorption Spectroscopy Measurements in Optically Dense Explosive Fireballs Using a Modeless Broadband Dye Laser, Appl. Spectrosc. 63 (2009) 1075-1080.

[20] M. Soo, N. Glumac, Ultraviolet absorption spectroscopy in optically dense fireballs using broadband secondharmonic generation of a pulsed modeless dye laser., Appl. Spectrosc. 68 (2014) 517-24.

[21] R.D. Cowan, G.H. Dieke, Self-Absorption of Spectrum Lines, Rev. Mod. Phys. 20 (1948) 418-455.

[22] H. Amamou, A. Bois, B. Ferhat, R. Redon, B. Rossetto, M. Ripert, Correction of the self-absorption for reversed spectral lines: application to two resonance lines of neutral aluminium, J. Quant. Spectrosc. Radiat. Transf. 77 (2003) 365-372.

[23] A.K. Pradhan, S.N. Nahar (2015), Atomic Astrophysics and Spectroscopy, Cambridge University Press.

[24] W.C. Marlow, Hakenmethode, Appl. Opt. 6 (1967) 1715-1724.

[25] J.M. Peuker, P. Lynch, H. Krier, N. Glumac, On AlO Emission Spectroscopy as a Diagnostic in Energetic Materials Testing, Propellants, Explos. Pyrotech. 38 (2013) 577-585.

[26] J. Kalman, D. Allen, N. Glumac, H. Krier, Optical Depth Effects on Aluminum Oxide Spectral Emissivity, J. Thermophys. Heat Transf. 29 (2015) 74-82. 
[27] R.A. Reed, V.S. Calia, Review of aluminum oxide rocket exhaust particles, $28^{\text {th }}$ Thermophys. Conf. (1993) AIAA paper 92-2819.

[28] P. Lynch, H. Krier, N. Glumac, Emissivity of Aluminum-Oxide Particle Clouds: Application to Pyrometry of Explosive Fireballs, J. Thermophys. Heat Transf. 24 (2010) 301-308.

[29] N. Glumac, H. Krier, T. Bazyn, R. Eyer, Temperature Measurements of Aluminum Particles Burning in Carbon Dioxide, Combust. Sci. Technol. 177 (2005) 485-511.

[30] C.M. Western, PGOPHER: A program for simulating rotational, vibrational and electronic spectra, J. Quant. Spectrosc. Radiat. Transf. 186 (2017) 221-242.

[31] A.T. Patrascu, S.N. Yurchenko, J. Tennyson, ExoMol molecular line lists - IX. The spectrum of AlO, Mon. Not. R. Astron. Soc. 449 (2015) 3613-3619.

[32] B. McBride, S. Gordon, Computer Program for Calculation of Complex Chemical Equilibrium Compositions and Applications, NASA RP-1311, NASA Lewis Research Center, Cleveland, OH, 1996.

[33] C.K. Law, A Simplified Theoretical Model for the Vapor-Phase Combustion of Metal Particles, Combust. Sci. Technol. 7 (1973) 197-212.

[34] A. Fontijn, W. Felder, J.J. Houghton, HTFFR kinetics studies. Temperature dependence of $\mathrm{Al} / \mathrm{O}_{2}$ and $\mathrm{AlO} / \mathrm{O}_{2}$ kinetics from 300 to 1700/1400 K, Symp. (Int.) Combust. 16 (1977) 871-879.

[35] A. Fontijn, W. Felder, HTFFR kinetics studies of $\mathrm{Al}+\mathrm{CO}_{2}-\mathrm{AlO}+\mathrm{CO}$ from 300 to $1900 \mathrm{~K}$, a non-Arrhenius reaction, J. Chem. Phys. 67 (1977) 1561-1569.

[36] T.A. Brzustowski, I. Glassman, Spectroscopic Investigation of Metal Combustion, H.G. Wolfhard, I. Glassman, L. Green (Eds.), Heterogeneous Combustion Vol. 15, Academic Press, New York, 1964, pp. 41-73.

[37] N.I. Poletaev, A.V. Florko, Spectral studies of the gas component of an aluminum dust flame, Combust. Explos. Shock Waves 44 (2008) 437-443.

[38] J.M. Bergthorson, S.D. Salusbury, P.E. Dimotakis, Experiments and modelling of premixed laminar stagnation flame hydrodynamics, J. Fluid Mech. 681 (2011) 340-369.

[39] G. Yu, C. Law, C. Wu, Laminar flame speeds of hydrocarbon + air mixtures with hydrogen addition, Combust. Flame 63 (1986) 339-347. 